\title{
A PERMANENCE THEOREM FOR SUMS OF SEQUENCE SPACES
}

\author{
A. K. SNYDER
}

\begin{abstract}
Let $/$ be the space of absolutely summable sequences. Using difficult functional analytic techniques Bennett proved that if $X$ is a separable FK space containing $\delta^{n}$ for all $n$ and if $\delta^{n} \rightarrow 0$ in $X+l$, then $l \subset X$. Bennett also asked whether the separability assumption can be dropped. Using an elementary invertibility criterion for Banach algebras, the present note gives a self-contained proof that if $z$ is a null sequence, $X$ is an FK space containing $\delta^{n}$ for all $n$, and $X+z l=l$, then $X=l$. This answers Bennett's question in the affirmative.
\end{abstract}

1. Introduction. In the process of examining properties of certain sequence spaces related. to summability theory, Bennett in [1] proved a difficult permanence type theorem about wedge spaces. A topological sequence space $X$ containing the sequences $\delta^{n}\left(\delta_{k}^{n}=0\right.$ for $\left.k \neq n, \delta_{n}^{n}=1\right)$ is a wedge space if $\delta^{n} \rightarrow 0$ in $X$. It is easy to see that the sum $X+Y$ of two FK spaces can be a wedge space without either $X$ or $Y$ being wedge. However, using difficult functional analytic techniques, Bennett proved the surprising result that if $X$ is a separable FK space containing $\delta^{n}$ for all $n$ and if $X+l$ is wedge, then $l \subset X$ (so $X$ is a wedge space). Here $l$ is the space of absolutely summable sequences. The purpose of the present note is to provide a more elementary proof of Bennett's theorem. More interesting is the fact that the proof allows the separability assumption to be dropped. The technique involves the examination of certain operators on $l$.

2. Preliminaries. Let $\omega$ denote the space of all complex sequences $x=\left\{x_{n}\right\}$. An $F K$ space is a subspace of $\omega$ which is a locally convex Fréchet space on which the coordinate functionals $\left\{x_{n}\right\} \rightarrow x_{n}$ are continuous. A $B K$ space is an FK space whose topology is normable. The topology of an FK space is generated by an appropriate sequence of seminorms or by a paranorm which ordinarily is a Fréchet combination of seminorms.

The following BK spaces will occur in the sequel:

$$
\begin{aligned}
& c_{0}=\left\{x \in \omega: \lim x_{n}=0\right\} \text { with }\|x\|_{\infty}=\sup \left|x_{n}\right|, \\
& l=\left\{x \in \omega:\|x\|_{1}=\sum\left|x_{n}\right|<\infty\right\}, \\
& l^{2}=\left\{x \in \omega:\|x\|_{2}=\left(\sum\left|x_{n}\right|^{2}\right)^{1 / 2}<\infty\right\} .
\end{aligned}
$$

Received by the editors January 23, 1984 and, in revised form, June 5, 1984. Presented at the 816th meeting of the American Mathematical Society, Anaheim, California, January 9, 1985.

1980 Mathematics Subject Classification. Primary 46A45; Secondary 40H05.

Key words and phrases. FK space, wedge space.

(c)1985 American Mathematical Society $0002-9939 / 85 \$ 1.00+\$ .25$ per page 
For $x, y \in \omega$ let $x y \in \omega$ be the sequence $\left\{x_{n} y_{n}\right\}$. For $x \in \omega, E \subset \omega$ let $x E=\{x y$ : $y \in E\}$. If $Y$ is an FK space (BK space) and $x \in \omega$, then $x Y$ is an FK space (BK space) identified as the quotient $Y / N, N$ the kernel of the map $y \rightarrow x y$. If $Y$ is a BK space with norm \|\|$_{Y}$ and if $x \in \omega$ with $x_{n} \neq 0$ for all $n$, let $\|t\|_{x Y}=\|t / x\|_{Y}$ where $1 / x=\left\{1 / x_{n}\right\}$.

Let $\phi$ be the linear span of the sequences $\delta^{n}$. If $X$ is an FK space containing $\phi$ and if $\delta^{n} \rightarrow 0\left(\delta^{n} \rightarrow 0\right.$ weakly) in $X$, then $X$ is a wedge (weak wedge) space.

Let $X$ and $Y$ be FK spaces whose topologies are given by paranorms $p$ and $q$, respectively. Then $X+Y=\{x+y: x \in X, y \in Y\}$ is an FK space with paranorm

$$
r(z)=\inf \{p(x)+q(y): x \in X, y \in Y, z=x+y\} .
$$

An analogous result holds for BK spaces.

A thorough treatment of the basic facts about FK spaces may be found in the first several chapters of [4]. See [3, p. 36] for a discussion of sums of spaces.

3. The main result. A theorem equivalent to a generalization of Bennett's wedge space theorem is proved. It is first established that the space involved may be assumed BK. The desired conclusion is then obtained using an elementary fact about Banach algebras applied to the operators on $l$.

THEOREM 1. Let $z \in c_{0}$. If $X$ is an FK space containing $\phi$ and if $X+z l=l$, then $X=l$.

Proof. It may be assumed that $z_{n} \neq 0$ for all $n$. Furthermore, $X$ may be assumed BK. To see the latter, choose $w \in c_{0}$ such that $\left\{z_{n} / w_{n}\right\} \in c_{0}$. Since $w_{n} \delta^{n} \rightarrow 0$ in $X+z l$, there exists $\left\{x^{n}\right\} \subset X \cap z l$ such that $\left\{x^{n}\right\}$ is bounded in $X$ and $\left\{w_{n} \delta^{n}-x^{n}\right\}$ is bounded in $z l$. Choose $u \in c_{0}, u_{n} \neq 0$ for all $n$, such that $\left\{p\left(u_{n} \delta^{n}\right)\right\} \in l$, where $p$ is a paranorm for $X \cap w l$. It follows that $u l \subset X \cap w l$. For $\lambda \in l$,

$$
w \lambda=\sum \lambda_{n} x^{n}+\sum \lambda_{n}\left(w_{n} \delta^{n}-x^{n}\right),
$$

the series converging in $w l$ since $z l \subset w l$. Let $Y=\left\{\sum \lambda_{n} x^{n}: \lambda \in l\right\}+u l$. Then $Y$ is a BK space containing $\phi, Y \subset X$, and $w l=Y+z l$. Therefore, $l=Y / w+z l / w$ and $Y / w$ is a $\mathrm{BK}$ space containing $\phi$. If $Y / w=l$, then $z l \subset w l=Y \subset X$, so $l=X+z l$ $=X$.

Assuming that $X$ is a BK space, the definition of the norm for $X+z l$ allows the choice of $\left\{x^{n}\right\} \subset X \cap z l$ such that

$$
\left\|x^{n}\right\|_{X}+\left\|\delta^{n}-x^{n}\right\|_{z l}-\left\|\delta^{n}\right\|_{X+z l} \rightarrow 0 .
$$

Then $\left\{x^{n}\right\}$ is bounded in $X$ and $\left\{\delta^{n}-x^{n}\right\}$ is bounded in $z l$.

For $\lambda \in z l$ let

$$
B \lambda=\sum \lambda_{n}\left(\delta^{n}-x^{n}\right)
$$

It is easy to see that $B$ is a bounded linear operator on $z l$.

Observe first that there exists $\alpha<1$ such that for $n$ large,

$$
\left\|\delta^{n}-x^{n}\right\|_{z l} \leqslant \alpha\left\|\delta^{n}\right\|_{X+z l}
$$


If not, there exists an increasing sequence $\left\{r_{n}\right\}$ of positive integers such that

$$
\left\|\delta^{r_{n}}-x^{r_{n}}\right\|_{z l}-\left\|\delta^{r_{n}}\right\|_{X+z l} \rightarrow 0 \text {. }
$$

Using (*), $x^{r_{n}} \rightarrow 0$ in $X$, so $x^{r_{n}} \rightarrow 0$ in $l$. But since $\left\{\delta^{r_{n}}-x^{r_{n}}\right\}$ is bounded in $z l$ and $z \in c_{0}$, it is easy to see that $\delta^{r_{n}}-x^{r_{n}} \rightarrow 0$ in $l$. Therefore, $\delta^{r_{n}} \rightarrow 0$ in $l$, a contradiction.

Since $\phi \subset X$, by adjusting finitely many members of $\left\{x^{n}\right\}$, it may be assumed that

$$
\left\|\delta^{n}-x^{n}\right\|_{z l} \leqslant \alpha\left\|\delta^{n}\right\|_{X+z l}
$$

for all $n$ where $\alpha<1$. But

$$
\left\|\delta^{n}\right\|_{X+z l} \leqslant\left\|\delta^{n}\right\|_{z l}=1 /\left|z_{n}\right|
$$

For $\lambda \in z l$,

$$
\|B \lambda\|_{z l} \leqslant \sum\left|\lambda_{n}\right|\left\|\delta^{n}-x^{n}\right\|_{z l} \leqslant \alpha \sum\left|\frac{\lambda_{n}}{z_{n}}\right|=\alpha\|\lambda\|_{z l}
$$

Therefore, $\|B\|<1$.

Finally, let $A=I-B$. Then $\|I-A\|<1$, so $A$ is invertible as an operator on $z l$. However, for $\lambda \in z l$,

$$
A \lambda=\lambda-\sum \lambda_{n}\left(\delta^{n}-x^{n}\right)=\sum \lambda_{n} x^{n} .
$$

The latter series converges in $X$ because $\left\{z_{n} x^{n}\right\}$ is bounded in $X$. Therefore,

$$
z l=A(z l) \subset X,
$$

so

$$
l=X+z l=X .
$$

An immediate consequence is a generalization of Bennett's wedge space theorem [1, Theorem 16].

THEOREM 2. If $X$ is an FK space containing $\phi$ and $X+l$ is a wedge space, then $l \subset X$.

Proof. According to [1, Theorem 1], there exists $z \in c_{0}$ such that $l / z \subset X+l$. Then $z(X \cap l / z)+z l=l$. By Theorem $1, z(X \cap l / z)=l$ so $l \subset l / z \subset X$.

4. Concluding remarks. The existence of theorems for weak wedge spaces analogous to Theorem 2 could not be determined in [1]. This work also failed to establish inclusion type theorems for weak wedge spaces similar to the wedge space inclusion theorem [1, Theorem 1]. The latter was used in the proof of Theorem 2. Partial results in the direction of weak wedge inclusion theorems were obtained by the present writer in [2]. Some new permanence and inclusion type theorems will be discussed elsewhere.

It is tempting to call an FK space $Y$ containing $\phi$ trivially embedded in an FK space $Z$, if for each FK space $X$ containing $\phi, X+Y=Z$ implies $X=Z$. Thus, Theorem 1 states that $z l$ is trivially embedded in $l$ for all $z \in c_{0}$. The writer is unable to determine whether $l$ can be replaced in Theorem 1 by, for instance, any BK space 
$Z$ with $\left\{\delta^{n}\right\}$ as an unconditional basis. More interesting is the fact that if $l$ is not trivially embedded in $l^{2}$, then Theorem 2 fails for weak wedge spaces.

\section{REFERENCES}

1. G. Bennett, $A$ new class of sequence spaces with applications in summability theory, J. Reine Angew. Math. 266 (1974), 49-75.

2. A. K. Snyder, Universal families for conull FK spaces, Trans. Amer. Math. Soc. 284 (1984), 389-399.

3. A. Wilansky, Topics in functional analysis, Lecture Notes in Math., Vol. 45, Springer-Verlag, 1967.

4. Summability through functional analysis, North-Holland, Amsterdam, 1984.

Department of Mathematics, Lehigh University, Bethlehem, Pennsylvania 18015 